\title{
Experimentally Validated Hopping-Transport Model for Energetically Disordered Organic Semiconductors
}

\author{
Tanvi Upreti®, ${ }^{1}$ Yuming Wang, ${ }^{2}$ Huotian Zhang, ${ }^{2}$ Dorothea Scheunemann, ${ }^{1}$ Feng Gao, ${ }^{2}$ and \\ Martijn Kemerink $\oplus^{1, *}$ \\ ${ }^{1}$ Department of Physics, Chemistry and Biology (IFM), Complex Materials and Devices, Linköping University, \\ 58183 Linköping, Sweden \\ ${ }^{2}$ Department of Physics, Chemistry and Biology (IFM), Biomolecular and Organic Electronics, Linköping \\ University, 58183 Linköping, Sweden
}

(Received 14 October 2019; published 17 December 2019)

\begin{abstract}
Charge transport in disordered organic semiconductors occurs by hopping of charge carriers between localized sites that are randomly distributed in a strongly energy-dependent density of states. Extracting disorder and hopping parameters from experimental data, such as temperature-dependent current-voltage characteristics, typically relies on parametrized mobility functionals that are integrated in a drift-diffusion solver. Surprisingly, the functional based on the extended Gaussian disorder model (eGDM) is extremely successful at this, despite it being based on the assumption of nearest neighbor hopping $(\mathrm{nnH})$ on a regular lattice. We here propose a variable-range hopping (VRH) model that is integrated in a freeware driftdiffusion solver. The mobility model is calibrated using kinetic Monte Carlo calculations and shows good agreement with the Monte Carlo calculations over the experimentally relevant part of the parameter space. The model is applied to temperature-dependent space-charge-limited current (SCLC) measurements of different systems. In contrast to the eGDM, the VRH model provides a consistent description of both $p$ - and $n$-type devices. We find a critical ratio of $a_{\mathrm{NN}} / \alpha$ (mean intersite distance:localization radius) of about three, below which hopping to non-nearest neighbors becomes important around room temperature and the eGDM cannot be used for parameter extraction. Typical (Gaussian) disorder values in the range 45-120 meV are found, without any clear correlation with photovoltaic performance, when the same active layer is used in an organic solar cell.
\end{abstract}

DOI: 10.1103/PhysRevApplied.12.064039

\section{INTRODUCTION}

Recent years have seen a considerable improvement in the field of organic photovoltaics (OPVs), where power conversion efficiencies exceeding $16 \%$ have been reached [1]. To improve upon the performance of such devices, further understanding of the underlying physics of charge transport becomes important, as the (transient) mobility of the generated charge carriers is one of the key factors that decides how efficiently the charges can escape recombination and be extracted [2-6]. Similar arguments can be made for the need to understand charge transport in other organic devices, including light-emitting diodes, fieldeffect transistors, and a range of mixed ionic-electronic devices. However, in the following, we focus on OPV materials.

Charge transport in these systems is commonly understood to occur via thermally activated tunneling, hopping, of charge carriers between localized sites. Due to the wide $\left(\gg k_{B} T\right)$ typically Gaussian or exponential distribution of

\footnotetext{
"martijn.kemerink@liu.se
}

site energies, the mobility becomes strongly dependent on temperature $T$, charge carrier density $n$, and electrostatic field $F$. Various approaches are proposed to calculate the mobility functional, $\mu(T, n, F)$, for hopping transport in disordered semiconductors. Seminal work by Bässler used kinetic Monte Carlo (kMC) simulations, to this end [2]. While the resulting Gaussian disorder model (GDM) rationalizes experimentally observed temperature dependencies, it only considers the low-density Boltzmann limit and shows discrepancies in the field dependence that are attributed to spatial correlations of the site energies $[7,8]$. The charge-density dependence of mobility beyond the Boltzmann limit is included in the so-called extended Gaussian disorder model (eGDM) [9]. Pasveer et al. [9] introduced a parametrization scheme for the corresponding mobility functional $\mu(T, n, F)$. Although these expressions are conveniently implemented in, e.g., drift-diffusion solvers, and are widely used, they are also heavily criticized [10-12]. A major point of criticism is the assumption of a regular cubic lattice and the use of a small and constant localization radius that enforces strict nearest neighbor hopping $(\mathrm{nnH})$ and gives rise to an erroneous 
field dependence, if the assumed conditions are not met [11]. Despite the fundamental criticism, it is, to date, unknown how severe, from a pragmatic experimentalist perspective, the errors are when one applies the eGDM parametrization to extract hopping and disorder parameters from experimental data. A strongly related problem is the absence of a complementary variable-range hopping (VRH) model for a Gaussian density of states that is of sufficient user-friendliness to be used in data analysis. A step in this direction was made in the works of Cottaar et al. and Nenashev et al., who derived analytical expressions for the functional $\mu(T, n)$ for VRH on a simple cubic lattice $[10,13]$.

Among the various experimental techniques that are used to characterize transient and quasi-static charge transport [5,14-18], space-charge-limited current (SCLC) [19] is one of the most commonly used methods to estimate the mobility of charge carriers and the underlying hopping and disorder parameters. Although a straightforward method from an experimental perspective, the interpretation of current density versus voltage $(j-V)$ curves obtained from (temperature-dependent) SCLC can be rather intricate $[20,21]$. In a recent paper, we reviewed the most commonly employed methods and sources of error and introduced an automated software to analyze SCLC(T) $j-V$ data using, among others, the $\mathrm{nnH}$ models discussed above [21]. While the widely used "slope 2 fitting" method clearly leads to erroneous results, the other models, with the exception of the correlated disorder model [8], typically lead to consistent results, regarding the energetic disorder of hole-only diodes. Electron transport is not addressed.

Here, we aim to propose and establish a VRH model for electrons and holes that is both physical and practical and that allows a consistent extraction of parameters from steady-state transport measurements, such as SCLC. The proposed model is calibrated against numerically exact kinetic Monte Carlo simulations of VRH and integrated in the open-source analysis tool described in Ref. [21], which can be downloaded as freeware [22]. The model is used to analyze temperature-dependent SCLC curves for electron- and hole-only devices from a variety of binary and ternary OPV materials. The investigated blends include both polymer:fullerene and polymer:nonfullerene blends, with power conversion efficiencies ranging from about $5 \%$ to about $14 \%$. While for a significant number of materials the nnH model can be applied, in the meaning of giving very similar hopping parameters to that of the VRH model, the major advantage of the VRH model is that it provides a consistent determination of the hopping parameters of all material systems. The extracted parameters allow predictions of whether $\mathrm{nnH}$ could be used alternatively. Especially for fullerene-containing layers, electron hopping to non-nearest neighbors cannot be ignored, leading to a significant underestimation of the energetic disorder in the lowest unoccupied molecular orbital (LUMO) level, if an (e)GDM analysis were to be performed.

\section{THEORY}

\section{A. Analytical variable-range hopping model}

In the case of intrinsic organic semiconductors, it is common to assume a Gaussian energy distribution for the localized states,

$$
g(\varepsilon)=\frac{N_{i}}{\sqrt{2 \pi} \sigma_{\mathrm{DOS}}} \exp \left[\frac{-\left(\varepsilon-\varepsilon_{0}\right)^{2}}{2 \sigma_{\mathrm{DOS}}^{2}}\right]
$$

Here, $\sigma_{\mathrm{DOS}}$ is the energetic disorder of the density of states (DOS), typically in the range $0.05-0.1 \mathrm{eV} ; \epsilon_{0}$ is the central energy of the highest occupied molecular orbital (HOMO) or the LUMO; and $N_{i}$ is the total site density of the randomly distributed localized states that is related to the mean intersite distance by $a_{\mathrm{NN}}=N_{i}^{-1 / 3}$.

The charge-hopping rates from site $i$ to site $j$ are typically described by either the Miller-Abrahams or Marcus expressions. Although the latter appears more physical, as it accounts in lowest order for the lattice deformation that is associated with the presence of a net charge on a piece of conjugated material (polaron formation), the resulting mobilities are typically similar for the two rates [13]. Since the Miller-Abrahams rates require one less parameter to be known, we choose to work with these rates, for which the hopping rate between sites $i$ and $j$ is given as

$$
v_{i j}=v_{0}^{\prime} \exp \left(\frac{-2 r_{i j}}{\alpha}\right) \exp \left(-\frac{\Delta E}{k_{B} T}\right),
$$

where $k_{B}$ is the Boltzmann constant, $\Delta E=\max \left(0, \varepsilon_{j}-\right.$ $\varepsilon_{i}-\vec{F} \cdot \vec{r}_{i j}$ ) with a localization radius $\alpha, F$ is the local electrostatic field vector, and $r_{i j}$ is the vector from $i$ to $j$. The prefactor $v_{0}^{\prime}=v_{0} \exp \left(2 a_{\mathrm{NN}} / \alpha\right)$ makes $v_{0}^{\prime}$ become the rate of downward hops to a nearest neighbor site and is introduced for consistency with mobility expressions from lattice models, and that we fix to $1 \times 10^{11} \mathrm{~s}^{-1}$, unless stated otherwise $[9,10,21]$. The prefactor $v_{0}$ is typically identified as the attempt-to-hop frequency.

Numerical solutions for the current density and chargecarrier mobility in finite systems, with rates given by Eq. (2) and site energies randomly drawn from Eq. (1), have been reported by various authors. Specifically, Pasveer et al. used a master-equation method to generate a parametrized mobility functional for hopping on a simple cubic lattice for $a_{\mathrm{NN}} / \alpha=10$ as [9]

$$
\mu(T, c, F) \approx \tilde{\mu}(T, c) f(T, F) .
$$

Expressions for the factors $\tilde{\mu}(T, c)$ and $f(T, F)$ are given within the Supplemental Material [23] for completeness. 
Below, we analyze a series of SCLC $(T)$ experiments using either the mobility functional, Eq. (3), which we refer to as the eGDM, or the mobilities from the analytical model that is introduced next.

The analytical VRH model is an extension of the MottMartens model, as reviewed by Coehoorn et al. [24], along the lines previously used by Zuo et al. for doped organic semiconductors $[25,26]$. The conductivity is given by a Miller-Abrahams-type expression,

$$
\sigma=\sigma_{0} \exp \left(-2 \alpha R^{*}-\frac{\varepsilon^{*}-\varepsilon_{F}}{k_{B} T}\right),
$$

where the conductivity prefactor is $\sigma_{0}=n \mu_{0}$, with $n$ as the charge-carrier density and $\mu_{0}$ as a mobility prefactor. Using a percolation argument, the conductivity is assumed to be governed by characteristic hops over a distance $R^{*}$, going from the Fermi energy, $\varepsilon_{F}$, to the transport energy $\varepsilon^{*} . R^{*}$ and $\varepsilon^{*}$ are related through density of states, Eq. (1), as

$$
B_{C}=\frac{4}{3} \pi\left(R^{*}\right)^{3} \int_{\varepsilon_{F}}^{\varepsilon^{*}} g(\varepsilon) d \varepsilon
$$

where $B_{C}$ is the critical number of bonds in the infinite percolating cluster. Maximizing Eq. (4) under the conditions set by Eq. (5) allows for variable-range hopping to occur. Although this will not be pursued further here, we notice that fixing $R^{*}=a_{\mathrm{NN}}$ leads to a nearest neighbor hopping model that rather accurately reproduces numerical simulations of the type performed by Pasveer et al. [9]

Because of the absence of a proper analytical theory to express the nonexponential terms in Eq. (4), i.e., the mobility prefactor, we make the ansatz

$$
\mu_{0}(T)=B\left(\frac{a_{\mathrm{NN}}^{2} v_{0}}{k_{B} T}\right)\left(\frac{k_{B} T}{\sigma_{\mathrm{DOS}}}\right)^{\lambda_{1}}\left(\frac{\alpha}{a_{\mathrm{NN}}}\right)^{\lambda_{2}} .
$$

This expression is similar to what was derived in Refs. $[10,13]$, except for the last term on the right-hand side, which has been introduced in analogy to the dimensionless energy scaling factor $\left(k_{B} T / \sigma\right)^{\lambda_{1}}$. Instead of the term $a_{\mathrm{NN}}^{2}$ in Eq. (6), one could expect a term $R^{2}$, which is the expectation value of the squared hopping distance. While this leads to a slightly improved agreement with $\mathrm{kMC}$ results for the simulations parametric in $T$ and $\sigma_{\mathrm{DOS}}$, it severely deteriorates the agreement for the simulations parametric in $a_{\mathrm{NN}}$ and $\alpha$, as shown in Figs. S1 and S2 within the Supplemental Material [23].

To include the effects of finite electric fields, we use the effective-temperature concept, as introduced by Marianer and Shklovskii [27] and later Baranovskii et al. [28]. In their work, it is proposed that the combined effect of electric field and temperature can be captured by replacing the lattice temperature, $T$, with an effective temperature for the charge-carrier distribution:

$$
T_{\mathrm{eff}}=\left[T^{2}+\left(\gamma \frac{q F \alpha}{k_{B}}\right)^{2}\right]^{1 / 2}
$$

where $\gamma \approx 0.67$. In the following, the mobility model Eqs. (4)-(7) will be referred to as the effective-temperature Gaussian disorder model (ET GDM).

Prior to applying the ET GDM model to experimental $j-V$ curves, a "universal" set of parameters, $\lambda_{1}, \lambda_{2}, B$, and $B_{c}$, is to be found. To this end, we phenomenologically calibrate the analytical model to mobilities obtained from numerically exact kinetic Monte Carlo simulations as a function of concentration and $F$ parametric in $\sigma, T$, $a_{\mathrm{NN}}$, and $\alpha$. Although the ET GDM model is physically transparent and leads to equations that are easily solvable by numerical means, integration in a drift-diffusion device solver that allows fitting of experimental data on reasonable timescales is not completely trivial. Hence, to make the methodology available to others, we implement it in the automated SCLC analysis program FitSCLC, which can be freely downloaded [21,22]. The same code can be used with the eGDM mobility functional and with a range of other analysis schemes.

\section{B. Kinetic Monte Carlo model}

Numerical kMC simulations are an established method to obtain numerically exact solutions of the transport problem posed by a finite density of interacting particles hopping in a landscape with positional and/or energetic disorder $[2,6,10,11]$. Here, we use a standard kMC scheme to generate reference mobilities as a function of chargecarrier concentration, disorder, temperature, localization radius, site density, and electric field. The program is similar to the one described in Ref. [6], except that it works on a random lattice, instead of a regular lattice, and that it allows for variable-range hopping by considering hops to a preset number of nearest neighbor sites, which is chosen to be sufficiently large that further increases no longer affect the mobility; typically 63-126 neighboring sites are included. Single-particle site energies are randomly selected from a Gaussian DOS [Eq. (1)]. Hopping rates are calculated from Eq. (2) and used as weight factors in the otherwise random selection of the hopping event in each time step. The waiting time for each time step is calculated as $\tau=-\left[\ln (r) / \Sigma_{v}\right]$, where $r$ is a random number drawn from a homogeneous distribution between zero and one and $\Sigma_{v}$ is the sum of the rates of all possible events. Onsite particle-particle interactions, preventing double site occupation, are included. 


\section{EXPERIMENT}

Electron and hole transport are analyzed using temperature-dependent space-charge-limited current measurements on single carrier devices based on a number of representative OPV materials. Specifically, the fullerenebased TQ1:PC 71 BM (poly\{[2,3-bis(3-octyloxyphenyl) -5,8-quinoxalinediyl]-2,5-thiophenediyl\}:[6,6]-Phenyl $\mathrm{C}_{71}$ butyric acid methyl ester) and nonfullerene-based PBDBT:IEICO-4F (poly(\{4,8-bis[5-(2-ethylhexyl)-2-thienyl]ben zo[1,2-b:4,5- $\left.b^{\prime}\right]$ dithiophene-2,6-diyl $\}-2,5$-thiophenediyl[5, 7-bis(2-ethylhexyl)-4,8-dioxo- $4 H, 8 H$-benzo[1,2-c:4,5-c'] dithiophene-1,3-diyl] $): 2,2^{\prime}-\left[\left(2 Z, 2^{\prime} Z\right)-(\{[4,4,9,9\right.$-tetr-akis (4-hexylphenyl)-4,9-dihydrosindaceno[1,2-b:5,6-b']dithi ophene-2,7-diyl]bis $\{4$-[(2-ethylhexyl)oxy]thiophene-5, 2-diyl\}\}bis(methanylylidene))bis(5,6-difluoro-3-oxo-2, 3 -dihydro-1 $H$-indene-2,1-diylidene) $]$ dimalononitrile) and PM6:Y6 (poly[(2,6-\{4,8-bis[5-(2-ethylhexyl-3-fluoro)thiophen-2-yl]benzo[1,2- $\left.b: 4,5-b^{\prime}\right]$ dithiophene $\left.\}\right)$-alt $-\left\{5,5-\left[1^{\prime}\right.\right.$, $3^{\prime}$-di-2-thienyl-5',7'-bis(2-ethylhexyl)benzo[ $\left[1^{\prime}, 2^{\prime}\right.$-c: $4^{\prime}, 5^{\prime}$ $\left.c^{\prime}\right]$ dithiophene-4,8-dione $\left.\left.]\right\}\right]: 2,2^{\prime}-\left(\left(2 Z, 2^{\prime} Z\right)-\{[12,13\right.$-bis (2-ethylhexyl)-3,9-diundecyl-12,13-dihydro[1,2,5]thiadia zolo[3,4-e]thieno[2, $\left./ 3^{\prime \prime}: 4^{\prime}, 5^{\prime}\right]$ thieno $\left[2^{\prime}, 3^{\prime}: 4,5\right]$ pyrrolo[3, $2-g]$ thieno[ $\left.2^{\prime}, 3^{\prime}: 4,5\right]$ thieno[3,2-b]indole-2,10-diyl]bis (methanylylidene)\}bis(5,6-difluoro-3-oxo-2,3-dihydro$1 H$-indene-2,1-diylidene))dimalononitrile) binary systems are investigated, along with the ternary PBDB-T: $\mathrm{PC}_{71} \mathrm{BM}$ : IEICO-4F system.

\section{A. Solution preparation}

PM6, PBDB-T, and IEICO-4F are purchased from Solarmer; $\mathrm{PC}_{71} \mathrm{BM}$ is purchased from the 1Material. Y6 is synthesized according to a procedure reported in the literature [29]. The solutions of binary PBDBT:IEICO-4F (1:1.5) and ternary PBDB-T:PC ${ }_{71}$ BM:IEICO$4 \mathrm{~F}(1: 0.7: 0.8)$ for the active layers are both prepared with concentrations of $20 \mathrm{mg} / \mathrm{mL}$ in total in a mixture of chloroform $(\mathrm{CF})$ and 1-chloronaphthalene (CN, 96 to 4 vol\%). PM6 and Y6 are dissolved in CF to a total concentration of $16 \mathrm{mg} / \mathrm{mL}$, with a 1 to 1.2 weight ratio, and $0.5 \% \mathrm{CN}$ (v:v, $\mathrm{CN}: \mathrm{CF}$ ) as an additive. The solution for the active layers is stirred at least for $5 \mathrm{~h}$.

\section{B. Device fabrication}

Hole-only devices are made with a configuration of glass/ITO/poly(3,4-ethylenedioxythiophene)-poly(styrenesulfonate) (PEDOT:PSS, $20 \mathrm{~nm}$ )/active layer $/ \mathrm{MoO}_{x}(8 \mathrm{~nm})$ $/ \mathrm{Al}(90 \mathrm{~nm})$, and the electron-only devices are made with a configuration of glass/ITO/polyethylenimine ethoxylated (PEIE, $2 \mathrm{~nm}) /$ active layer/ $\operatorname{LiF}(0.6 \mathrm{~nm}) / \mathrm{Al}(90 \mathrm{~nm})$. The ITO-coated glasses are boiled in a mixture of deionized water, ammonium hydroxide $(25 \%)$, and hydrogen peroxide $(28 \%, 5: 1: 1 \mathrm{vol})$ at $80^{\circ} \mathrm{C}$ for $15 \mathrm{~min}$ for cleaning.
PEDOT:PSS (Baytron P VP Al 4083, diluted with deionized water $1: 1 \mathrm{vol}$ ) is spin-coated onto the ITO glasses at $3000 \mathrm{rpm}$ for $40 \mathrm{~s}$, followed by annealing at $150^{\circ} \mathrm{C}$ for $10 \mathrm{~min}$. PEIE, dissolved in isopropyl alcohol (0.05 wt\%), is spin-coated onto the ITO glasses at $4000 \mathrm{rpm}$ for $30 \mathrm{~s}$, followed by annealing at $100^{\circ} \mathrm{C}$ for $10 \mathrm{~min}$. The $\mathrm{MoO}_{x} / \mathrm{Al}$ and $\mathrm{LiF} / \mathrm{Al}$ layers are deposited by thermal evaporation through a shadow mask. The active layers are deposited by spin-coating at $2500 \mathrm{rpm}$ for $60 \mathrm{~s}$, followed by annealing at $100^{\circ} \mathrm{C}$ for $10 \mathrm{~min}$.

\section{Electrical characterization}

Temperature-dependent SCLC measurements are performed inside a Janis cryogenic probe station under vacuum (approximately $10^{-5}$ mbar). Care is taken that $j-V$ curves recorded before and after the temperature sweep are essentially identical.

\section{RESULTS}

\section{A. Calibration and comparison of the analytical model with $\mathrm{kMC}$}

Before using the analytical VRH model to extract parameters from experimental data, we need to identify the values of the parameters for the mobility prefactor, Eq. (6). To this end, mobility is studied as a function of concentration and electric field, which is parametric in $\sigma_{\mathrm{DOS}}, T$, $a_{\mathrm{NN}}$, and $\alpha$ (see Fig. 1). The mobilities obtained with the exact kMC model (symbols) are globally fitted with the analytical model (lines) to determine the parameters $\lambda_{1}$, $\lambda_{2}, B$, and $B_{c}$. We find $B_{c} \approx 2.7$, in line with results previously reported in the literature $[10,30]$. The optimal values of $\lambda_{1}$ and $\lambda_{2}$ are 0 and 0.5 , respectively, but we should stress that changes in these values only lead to minor, mostly quantitative, changes that can be compensated by changing $B$.

Irrespective of the parameter values used, the mobility is constant at low concentrations and then increases in a power law fashion, which is in line with previous experimental results [31,32] and consistent with analytical [12] and numerical treatments of the Gaussian disorder model [9]. In the entire parameter space, the difference between the numerical and analytical models is well within a factor two, which is reasonable in view of typical sample-tosample variations encountered experimentally, with maximum variations occurring at low temperatures. While it is possible to improve the agreement in one panel, doing so would deteriorate agreement in another and the given parameters reflect a broad global optimum. All in all, the analytical model seems to capture the key traits of the quasi-atomistic simulations in a physically transparent manner, with sufficient accuracy to be used in experimental analysis. 

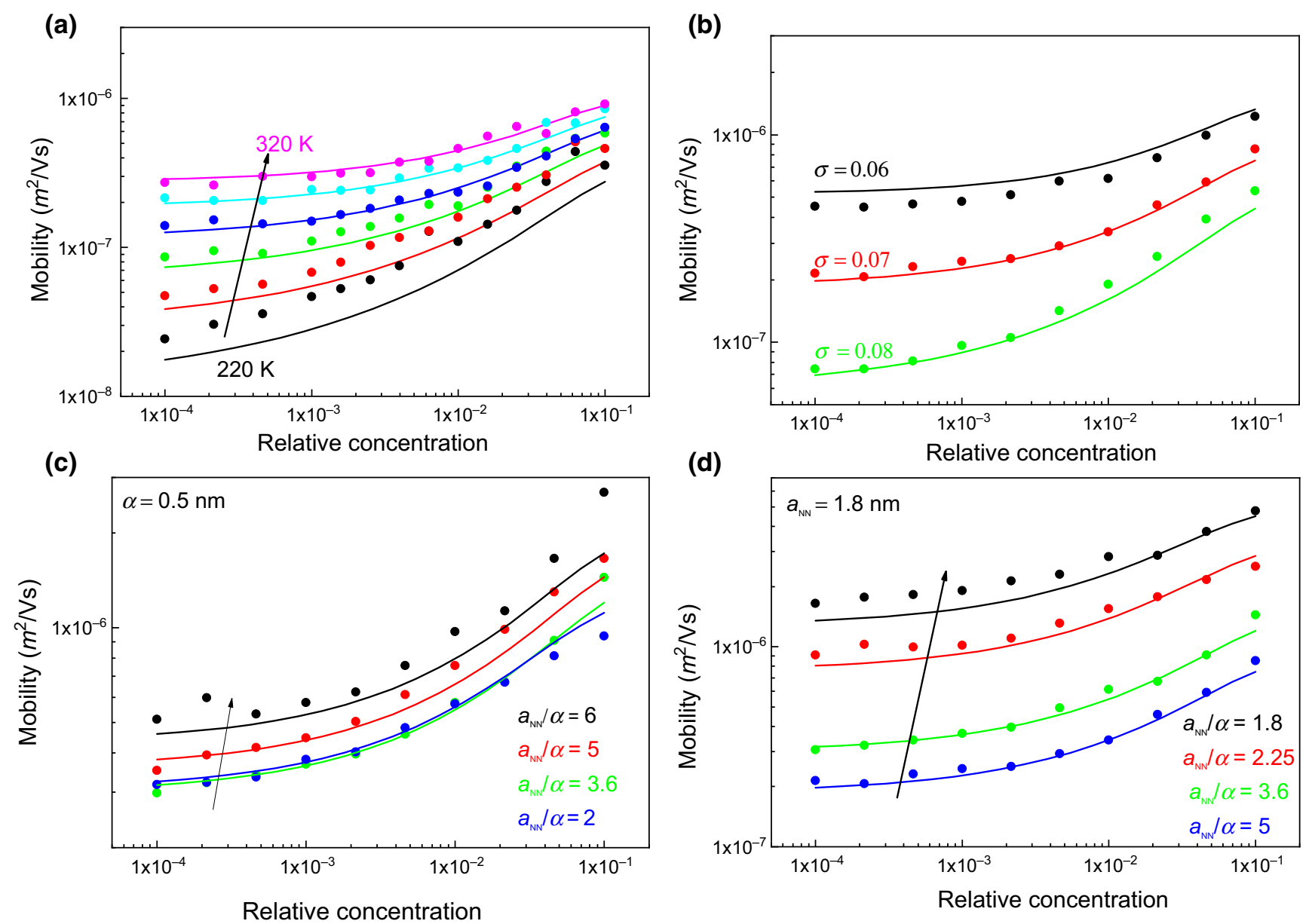

FIG. 1. Analytical model (lines) compared with kMC simulations (symbols) for charge-carrier mobility as a function of concentration parametric in temperature (a), disorder (b), mean intersite distance $a_{\mathrm{NN}}$ (c), and localization radius $\alpha$ (d). Parameters in the mobility calculation are $\sigma_{\mathrm{DOS}}=70 \mathrm{meV}, T=300 \mathrm{~K}, a_{\mathrm{NN}}=1.8 \mathrm{~nm}$, and $\alpha=0.36 \mathrm{~nm}$, unless indicated otherwise. Optimal parameters in the analytical model are $\lambda_{1}=0 \lambda_{2}=0.5, B=3$, and $B_{c}=2.7$ and are used throughout the text.

Figures 1(c) and 1(d) show the variation of mobility as a function of $a_{N N}$ and $\alpha$. In interpreting these results, the mobility prefactor $v_{0}^{\prime}=v_{0} \exp \left(2 a_{\mathrm{NN}} / \alpha\right)$, which is kept constant at $v_{0}^{\prime}=1 \times 10^{11} \mathrm{~s}^{-1}$, should be kept in mind. Specifically, the mobility increase with localization radius in Fig. 1(d) does not reflect the more or less trivial (exponential) dependence of $\mu$ on $\alpha$, but instead reflects the easier long-range percolation due to variable-range hopping; within the limit of strict nearest neighbor hopping, the plotted mobility would be independent of $\alpha$. Notably, the distinct dependence of the mobility on $a_{\mathrm{NN}}$ [Fig. 1(c)] and $\alpha$ stems, to a large degree, from the nonexponential terms in the mobility, as can be seen from the comparison with calculations for $\lambda_{2}=0$ in Fig. S1 within the Supplemental Material [23].

Shklovskii et al. [27] and Nenashev et al. [11] argue that the localization length $\alpha$, and not the typical intersite distance $a_{\mathrm{NN}}$, is the decisive length scale that determines the field dependence of mobility. In the limit of strict nearest neighbor hopping, as considered by Pasveer et al., this distinction becomes irrelevant [9]. In Fig. 2, we find good agreement between the field dependence of the mobility, as obtained by kMC, and the analytical model using the same parameters as those in Fig. 1. In view of the above, the apparent dependence of the field dependence of the mobility on $a_{\mathrm{NN}}$ in Fig. 2(c) may appear to be counterintuitive. However, although the effective temperature [Eq. (7)] does not depend on $a_{\mathrm{NN}}$ or $\sigma_{\mathrm{DOS}}$, cf. Fig. 2(b), the dependence of $\mu$ on the (effective) temperature does depend on these parameters, rationalizing the different high-field trends in Fig. 2.

\section{B. Analysis of experimental SCLC data}

Figure 3 shows the analysis of hole- and electrononly devices of the amorphous polymer:fullerene system TQ1:PCBM, using mobilities from both the eGDM and ET GDM models. For the hole-only devices, there is no noticeable difference in fit quality and, concomitantly, the extracted disorder values of $\sigma_{\mathrm{HOMO}}=85$ and $78 \mathrm{meV}$ for 

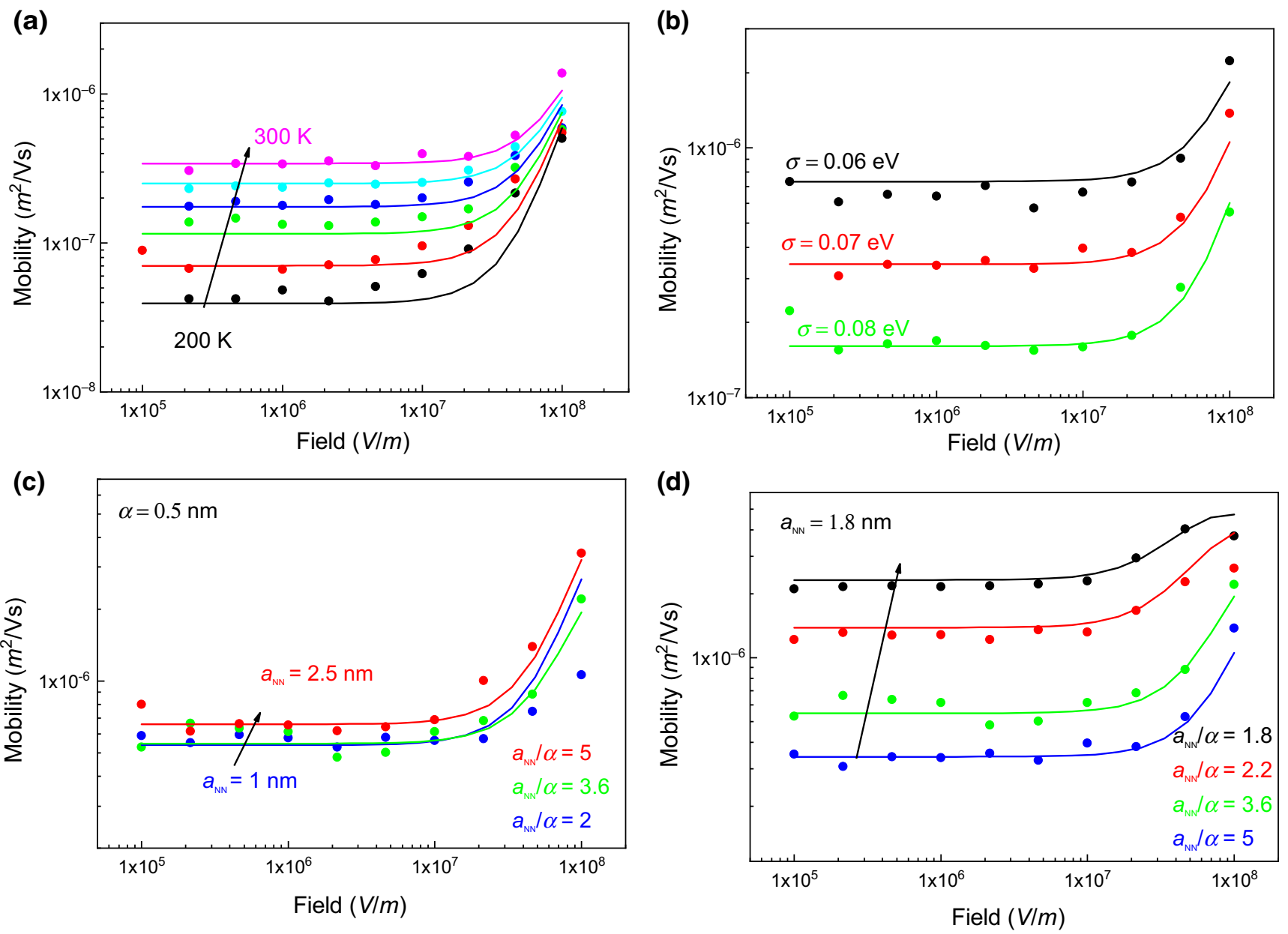

FIG. 2. Analytical model (lines) calibrated with kMC (symbols) for mobility as a function of field with the concentration kept at $1 \times 10^{-2}$, parametric in temperature (a), disorder (b), mean intersite distance $a_{\mathrm{NN}}$ (c), and localization radius $\alpha$ (d). Parameters in the mobility calculation are $\sigma_{\mathrm{DOS}}=70 \mathrm{meV}, T=300 \mathrm{~K}, a_{\mathrm{NN}}=1.8 \mathrm{~nm}$, and $\alpha=0.36 \mathrm{~nm}$, unless indicated otherwise.

the eGDM and ET GDM models, respectively, are rather similar. An overview of all fitted disorder and hopping parameters of all systems investigated is shown in Table S1 within the Supplemental Material [23]. For the electrononly devices, the situation is markedly different, and the eGDM (blue dashes) clearly fails to produce an acceptable fit, whereas the ET GDM (solid red) does. This is reflected in the dissimilar disorder values of $\sigma_{\mathrm{LUMO}}=100$ and $115 \mathrm{meV}$ for the eGDM and ET GDM models, respectively. The underlying reason for the failure of the eGDM is apparent from the small value of the ratio $a_{\mathrm{NN}} / \alpha=2.0$, which indicates that hopping beyond the nearest neighbor is already important around room temperature. As variable-range hopping is known to suppress the temperature dependence of mobility, any model that only accounts for nearest neighbor hopping will (try to) compensate with a low(er) energetic disorder, which also leads to a lower temperature dependence $[9,33]$. In this context, it is important that the fitted ET GDM value of $\sigma_{\text {LUMO }}=115 \mathrm{meV}$ is within experimental uncertainty equal to the value of about
$120 \mathrm{meV}$ found from a detailed analysis of dispersive ultrafast charge transport in the same system $[5,6]$.

It is in this context instructive to briefly address analysis of the data in Fig. 3 using the Murgatroyd model, in which an empirical field enhancement of mobility is integrated into an analytical expression for space-chargelimited transport; full details are given in Sec. 5 and Fig. S3 within the Supplemental Material [23] and Ref. [21]. Although this procedure leads to excellent fits of both sets of $j-V$ data, it yields $\sigma_{\mathrm{HOMO}}=87 \mathrm{meV}$ and $\sigma_{\mathrm{LUMO}}=$ $82 \mathrm{meV}$. In other words, it leads to similar or even larger deviations from the actual disorder values than those of the eGDM, which is not surprising in view of the fact that the temperature-dependent zero-field mobility that is extracted from the Murgatroyd fit is analyzed using the classical GDM model.

In view of the recent surge in nonfullerene acceptor materials, and the concomitant rise in conversion efficiencies in the OPV field, it is important that the proposed ET GDM model also provides a consistent description 

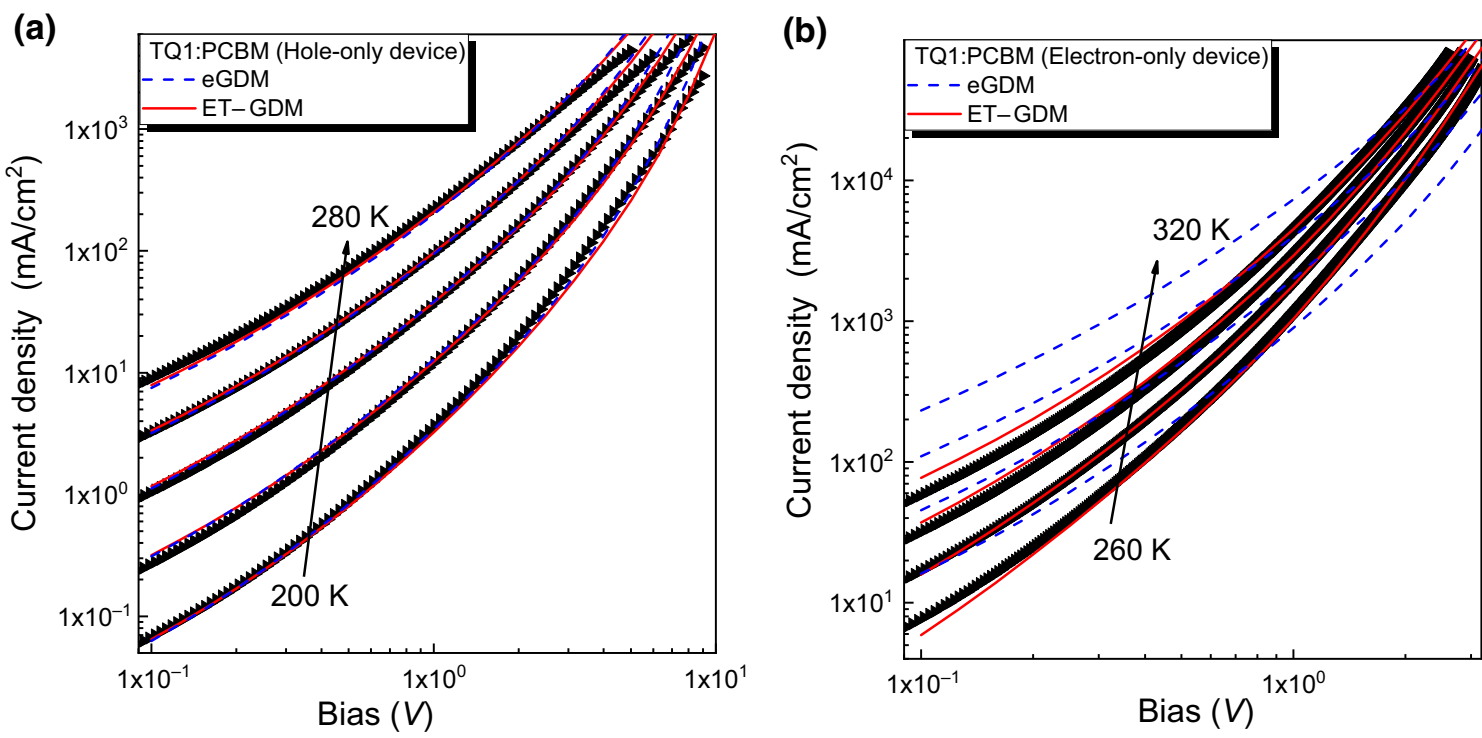

FIG. 3. Experimental $j-V$ curves (symbols) and numerical drift-diffusion model fits using mobilities from the eGDM (blue dashed lines) and ET GDM (solid red lines) models for TQ1:PCBM hole-only (a) and electron-only (b) devices. Extracted disorder and hopping parameters are listed in Table S1 in Sec. 3 within the Supplemental Material [23].

of charge transport in these systems. In addition, reliable disorder values for these compounds are rare.

One of the currently best performing binary systems is the PM6:Y6 system, reaching power conversion efficiencies (PCEs) in excess of $15 \%$ in optimized devices [29]. In our hands, this system has a PCE of around 14\%, see Table S2 within the Supplemental Material [23]. Figure 4 shows the analysis of SCLC data for hole- (panel a) and electron-only (panel b) devices of this blend. For these devices, there is no significant difference in the fit quality nor in the extracted disorder and hopping parameters, cf. Table S1 within the Supplemental Material. This equivalence is consistent with the $a_{\mathrm{NN}} / \alpha$ ratios of around five that are found from the ET GDM fit, indicating that around room temperature hopping is predominantly to nearest neighbors.

Intuitively, on the basis of the low energy loss in this OPV system, one might expect a relatively small energetic disorder [29,34]. However, we find $\sigma_{\mathrm{HOMO}}=89 \mathrm{meV}$ and $\sigma_{\text {LUMO }}=71 \mathrm{meV}$, which, in a quasi-equilibrium view of OPV devices, would correspond to a disorder-induced voltage loss in excess of $0.2 \mathrm{~V}$ [35]. Although this is beyond the scope of the present work, we note that the high PCE values observed for this system are, therefore, unlikely to be related to a suppressed energetic disorder.

Although the underlying mechanisms are incompletely understood, ternary blends of two donors and one acceptor, or vice versa, are proven to be a viable route to PCE values that exceed those of the corresponding binary compounds [36,37]. In a recent work, some of us argued that the quasi-linear composition dependence of the opencircuit voltage, which is crucial for the improved PCE of ternary systems, is quantitatively related to the finite width of the density of states of the constituent materials and its variation with composition [38,39]. It is therefore of interest to also test the mobility model on a recent ternary blend consisting of one donor and two acceptors. Temperature-dependent charge transport is examined for the PBDB-T:IEICO-4F:PCBM system, using two different recipes (solvent + additive, see Sec. III). In addition, the complementary binary PBDB-T:IEICO-4F systems are investigated [40]. Figure 5 shows a representative example; the SCLC data for the other combinations are shown in Sec. 5 within the Supplemental Material [23].

As for the example in Fig. 5, virtually all of the singlecarrier SCLC data for this material system are equally well fitted by either of the two mobility models. This optical similarity notwithstanding, the disorder values obtained by the eGDM can differ markedly from those obtained with the ET GDM model. Deviations between the two models clearly correlate with the value of the $a_{N N} / \alpha$ ratio, as shown in Fig. S3 within the Supplemental Material [23], with deviations exceeding $10 \mathrm{meV}$ occurring around $a_{\mathrm{NN}} / \alpha=3$ and below. Hence, as for the Murgatroyd fit discussed in the context of Fig. 3 above, an "optically" good fit does not guarantee an appropriate model is used.

A factor that can hamper the accurate parameter extraction from SCLC curves are charge-carrier traps that may, for instance, be induced by nanoscopic water clusters and that show up as a "hump" in the $j-V$ curve, which cannot, without further additions, be reproduced by a driftdiffusion model with either of the investigated mobility expressions [41]. Alternatively, morphological dead ends may act as charge traps that suppress the current (increase) 

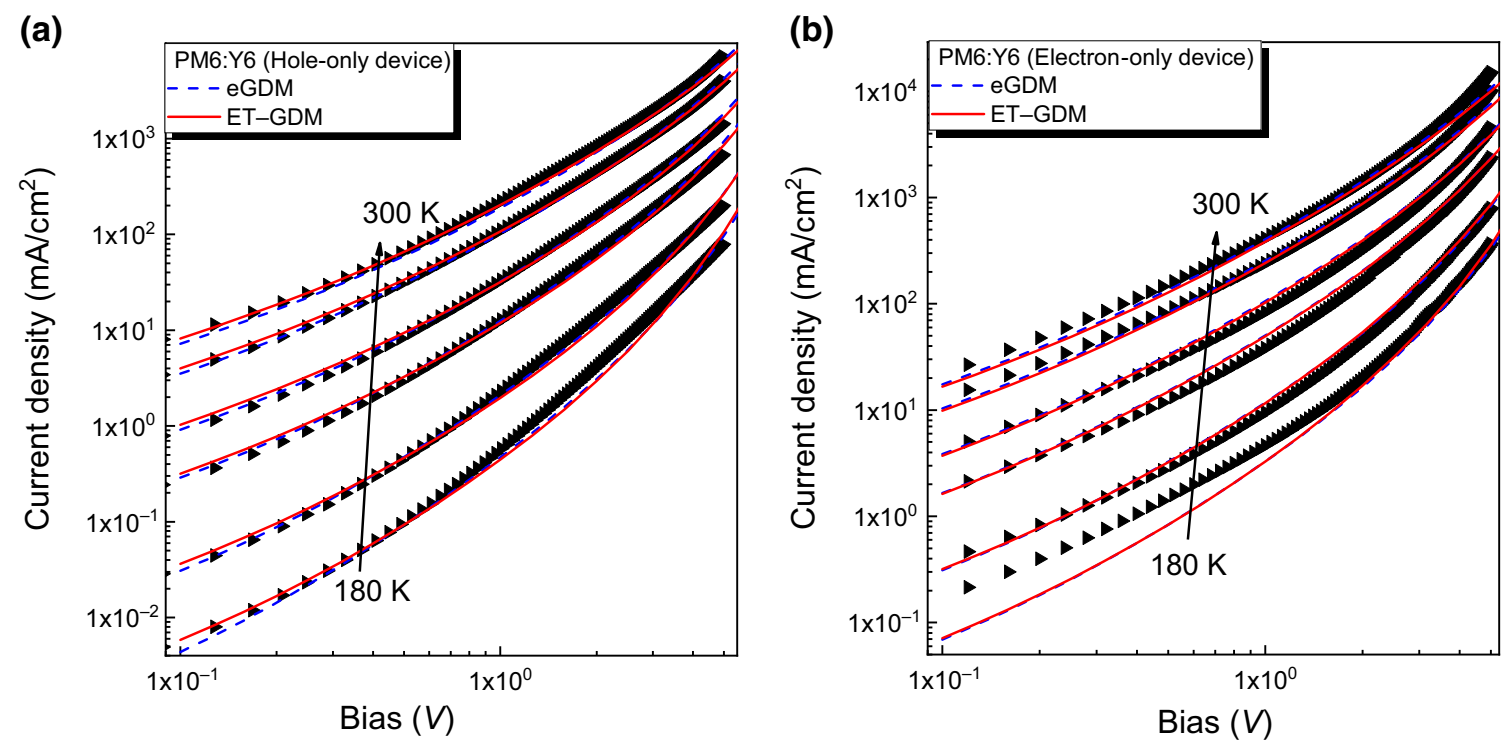

FIG. 4. Experimental $j$ - $V$ curves (symbols) and numerical drift-diffusion model fits using mobilities from the eGDM (blue dashed lines) and ET GDM (solid red lines) models for PM6:Y6 hole-only (a) and electron-only (b) devices. Extracted disorder and hopping parameters are listed in Table S1 in Sec. 3 within the Supplemental Material [23].

at higher fields $[42,43]$. Although not visible in Fig. 5, the PBDB-T:IEICO-4F system has a clear propensity to form various types of traps, especially for electrons, cf. Figs. S5-S7 within the Supplemental Material [23]. Although this does not correlate with an increase in photovoltaic performance (Table S2 within the Supplemental Material [23], we note that the addition of PCBM to the layer deposited from chloroform seems to suppress the traps. A similar effect of PCBM was observed in Ref. [44]. A hand-waving argument for this behavior would be that

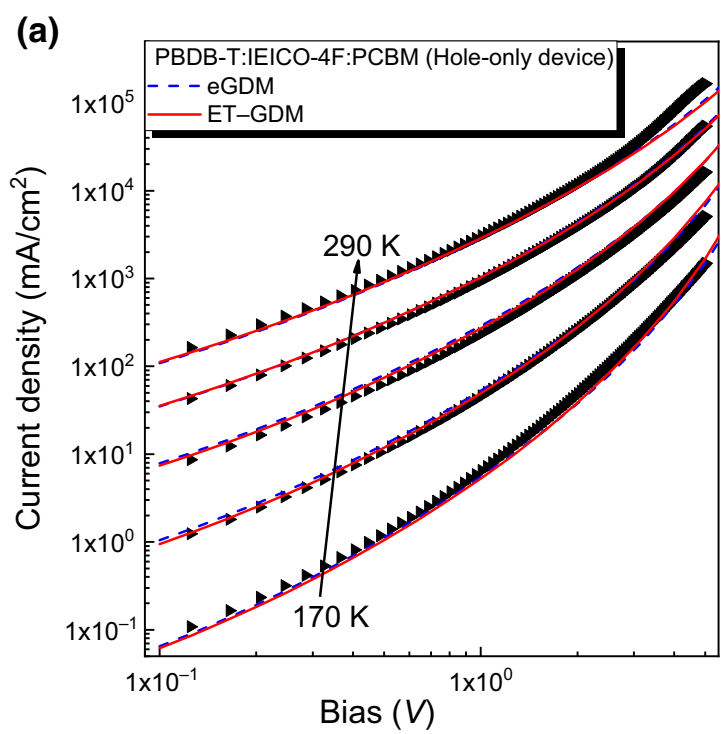

PCBM occupies the voids that originally are occupied by water and thereby gives a more compact morphology with a lower concentration of traps $[41,45]$.

In view of the uncertainties induced by charge trapping in this system, we refrain from a detailed interpretation of specific changes of parameters in response to changes in processing or composition. We do, however, notice that the disorder parameters of this system, as for the other systems examined here, are rather unremarkable in the range of 50-80 meV, with $\sigma_{\text {LUMO }}$ typically being somewhat smaller

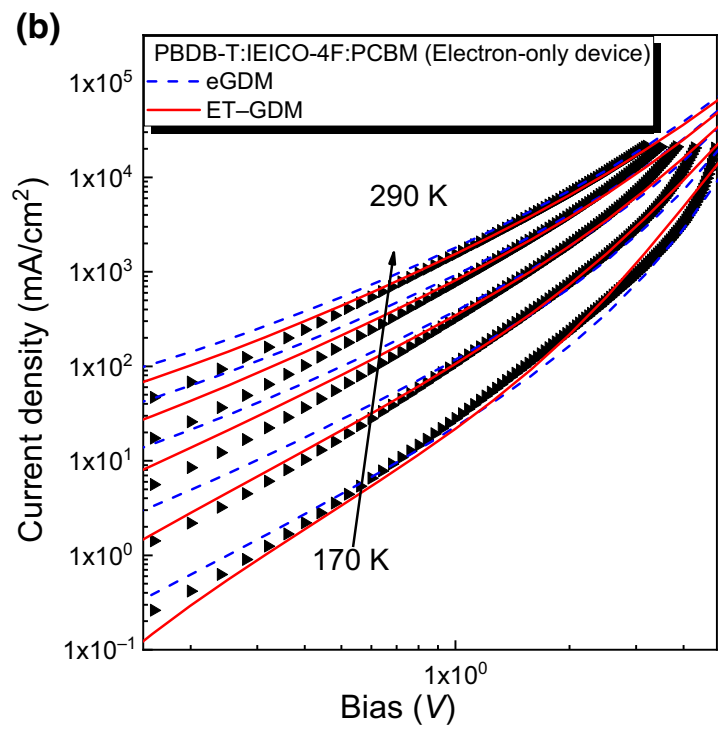

FIG. 5. Experimental $j-V$ curves (symbols) and numerical drift-diffusion model fits using mobilities from the eGDM (blue dashed lines) and ET GDM (solid red lines) models for PBDB-T:IEICO-4F:PCBM (CF + 4\% CN) hole-only (a) and electron-only (b) devices. Extracted disorder and hopping parameters are listed in Table S1 in Sec. 3 within the Supplemental Material [23]. 
than that of $\sigma_{\mathrm{HOMO}}$ and substantially smaller than what is typically found for PCBM, here and elsewhere [5,6,46]. We finally note that the addition of PCBM to the binary system does not seem to lead to an appreciable increase in $\sigma_{\text {LUMO }}$, which, at least in part, can be rationalized by the fact that the LUMO level of IEICO-4F lies below that of PCBM, so that electron transport will predominantly take place through the IEICO-4F subphase [40].

\section{CONCLUSIONS}

We present a variable-range hopping model for electron and hole transport in energetically disordered organic semiconductors. Whereas the exponential terms in the model are based on previous literature, the algebraic mobility prefactor is heuristically obtained and calibrated by direct comparison between the analytical model and kinetic Monte Carlo simulations over a relevant range of parameters. Good agreement between the two models is obtained. The analytical mobility expressions are subsequently integrated in a freeware drift-diffusion solver that can be used for automated fitting of temperature-dependent space-charge-limited current data $[21,22]$.

Analysis of experimental current-voltage curves shows that the proposed model can adequately describe electron and hole transport in a wide variety of organic semiconductor blends, which are used as the active layer in typical bulk heterojunction organic solar cells. Specifically, transport in binary and ternary blends based on both fullerene and nonfullerene acceptors can consistently be described. The latter is in contrast to the extended Gaussian disorder model based on nearest neighbor hopping on a regular lattice that gives only reliable results around room temperature, when the ratio of the typical intersite distance and the localization radius is larger than about three.

Disorder values of all investigated materials fall in the range of $50-90 \mathrm{meV}$, with the noticeable exception of PCBM $\left(\sigma_{\text {LUMO }}=115 \mathrm{meV}\right)$, without any clear correlation to OPV performance of the particular blend. This work does, however, show that nonfullerene acceptors that enable PCEs over $14 \%$ have significant (several $k_{B} T$ ) energetic disorder. Hence, the superior performance of these materials is unlikely to be due to suppressed energetic disorder. At the same time, these "modern" materials should, therefore, show the same dispersive ultrafast transport and concomitant slow carrier relaxation as that previously found for fullerene acceptors and polymeric donor and acceptor materials [47]. This will be a topic of future work.

\section{ACKNOWLEDGMENTS}

We gratefully acknowledge funding by Vetenskapsrådet, project “OPV2.0." D.S acknowledges funding from the European Union's Horizon 2020 research and innovation program under Marie Skłodowska-Curie Grant No.
799477 - HyThermEl. We thank J. Yuan for providing the Y6 material.

[1] B. Fan, D. Zhang, M. Li, W. Zhong, Z. Zeng, L. Ying, F. Huang, and Y. Cao, Achieving over 16\% efficiency for single-junction organic solar cells, Sci. China Chem. 62, 746 (2019).

[2] H. Bässler, Charge transport in disordered organic photoconductors a monte carlo simulation study, Phys. Status Solidi (b) 175, 15 (1993).

[3] C. Deibel, A. Wagenpfahl, and V. Dyakonov, Influence of charge carrier mobility on the performance of organic solar cells, Phys. Status Solidi Rapid Res. Lett. 2, 175 (2008).

[4] I. A. Howard, F. Etzold, F. Laquai, and M. Kemerink, Nonequilibrium charge dynamics in organic solar cells, Adv. Energy Mater. 4, 1301743 (2014).

[5] A. Melianas, V. Pranculis, A. Devižis, V. Gulbinas, O. Inganäs, and M. Kemerink, Dispersion-dominated photocurrent in polymer:Fullerene solar cells, Adv. Funct. Mater. 24, 4507 (2014).

[6] A. Melianas, F. Etzold, T. J. Savenije, F. Laquai, O. Inganäs, and M. Kemerink, Photo-generated carriers lose energy during extraction from polymer-fullerene solar cells, Nat Commun. 6, 8778 (2015).

[7] S. V. Novikov, D. H. Dunlap, V. M. Kenkre, P. E. Parris, and A. V. Vannikov, Essential Role of Correlations in Governing Charge Transport in Disordered Organic Materials, Phys. Rev. Lett. 81, 4472 (1998).

[8] M. Bouhassoune, S. L. M. van Mensfoort, P. A. Bobbert, and R. Coehoorn, Carrier-density and field-dependent charge-carrier mobility in organic semiconductors with correlated Gaussian disorder, Org. Electron. 10, 437 (2009).

[9] W. F. Pasveer, J. Cottaar, C. Tanase, R. Coehoorn, P. A. Bobbert, P. W. M. Blom, D. M. de Leeuw, and M. A. J. Michels, Unified Description of Charge-Carrier Mobilities in Disordered Semiconducting Polymers, Phys. Rev. Lett. 94, 206601 (2005).

[10] A. V. Nenashev, F. Jansson, J. O. Oelerich, D. Huemmer, A. V. Dvurechenskii, F. Gebhard, and S. D. Baranovskii, Advanced percolation solution for hopping conductivity, Phys. Rev. B 87, 235204 (2013).

[11] A. V. Nenashev, J. O. Oelerich, A. V. Dvurechenskii, F. Gebhard, and S. D. Baranovskii, Fundamental characteristic length scale for the field dependence of hopping charge transport in disordered organic semiconductors, Phys. Rev. B 96, 035204 (2017).

[12] S. D. Baranovskii, Mott lecture: Description of charge transport in disordered organic semiconductors: Analytical theories and computer simulations, Phys. Status Solidi (a) 215, 1700676 (2018).

[13] J. Cottaar, L. J. A. Koster, R. Coehoorn, and P. A. Bobbert, Scaling Theory for Percolative Charge Transport in Disordered Molecular Semiconductors, Phys. Rev. Lett. 107, 136601 (2011).

[14] P. N. Murgatroyd, Theory of space-charge-limited current enhanced by Frenkel effect, J. Phys. D Appl. Phys. 3, 151 (1970).

[15] J. Lorrmann, M. Ruf, D. Vocke, V. Dyakonov, and C. Deibel, Distribution of charge carrier transport properties 
in organic semiconductors with Gaussian disorder, J. Appl. Phys. 115, 183702 (2014).

[16] M. Mesta, C. Schaefer, J. de Groot, J. Cottaar, R. Coehoorn, and P. A. Bobbert, Charge-carrier relaxation in disordered organic semiconductors studied by dark injection: Experiment and modeling, Phys. Rev. B 88, 174204 (2013).

[17] G. Juška, K. Arlauskas, M. Viliūnas, and J. Kočka, Extraction Current Transients: New Method of Study of Charge Transport in Microcrystalline Silicon, Phys. Rev. Lett. 84, 4946 (2000).

[18] J. Cabanillas-Gonzalez, T. Virgili, A. Gambetta, G. Lanzani, T. D. Anthopoulos, and D. M. de Leeuw, Photoinduced Transient Stark Spectroscopy in Organic Semiconductors: A Method for Charge Mobility Determination in the Picosecond Regime, Phys. Rev. Lett. 96, 106601 (2006).

[19] A. Melianas, V. Pranculis, Y. Xia, N. Felekidis, O. Inganäs, V. Gulbinas, and M. Kemerink, Photogenerated carrier mobility significantly exceeds injected carrier mobility in organic solar cells, Adv. Energy Mater. 7, 1602143 (2017).

[20] J. C. Blakesley, F. A. Castro, W. Kylberg, G. F. A. Dibb, C. Arantes, R. Valaski, M. Cremona, J. S. Kim, and J.-S. Kim, Towards reliable charge-mobility benchmark measurements for organic semiconductors, Org. Electron. 15, 1263 (2014).

[21] N. Felekidis, A. Melianas, and M. Kemerink, Automated open-source software for charge transport analysis in single-carrier organic semiconductor diodes, Org. Electron. 61, 318 (2018).

[22] M. Kemerink, FitSCLC. (2018). https://github.com/ mkemerink/FitSCLC

[23] See the Supplemental Material at http://link.aps.org/supp lemental/10.1103/PhysRevApplied.12.064039 for further details on parametrization, modeling, experiments, and fitting parameters, as well as analyses of systems not shown in the main text.

[24] R. Coehoorn, W. F. Pasveer, P. A. Bobbert, and M. A. J. Michels, Charge-carrier concentration dependence of the hopping mobility in organic materials with Gaussian disorder, Phys. Rev. B 72, 155206 (2005).

[25] G. Zuo, H. Abdalla, and M. Kemerink, Impact of doping on the density of states and the mobility in organic semiconductors, Phys. Rev. B 93, 235203 (2016).

[26] G. Zuo, H. Abdalla, and M. Kemerink, Conjugated polymer blends for organic thermoelectrics, Adv. Electron. Mater. 5, 1800821 (2019).

[27] S. Marianer and B. I. Shklovskii, Effective temperature of hopping electrons in a strong electric field, Phys. Rev. B 46, 13100 (1992).

[28] S. D. Baranovskii, B. Cleve, R. Hess, and P. Thomas, Effective temperature for electrons in band tails, J. Non-Crystalline Solids 164-166, 437 (1993).

[29] J. Yuan, Y. Zhang, L. Zhou, G. Zhang, H.-L. Yip, T.-K. Lau, X. Lu, C. Zhu, H. Peng, P. A. Johnson, M. Leclerc, Y. Cao, J. Ulanski, Y. Li, and Y. Zou, Single-Junction organic solar cell with over $15 \%$ efficiency using fused-ring acceptor with electron-deficient core, Joule 3, 1140 (2019).

[30] B. I. Shklovskii and A. L. Efros, Electronic Properties of Doped Semiconductors (Springer Verlag, Heidelberg, 1984).
[31] C. Tanase, E. J. Meijer, P. W. M. Blom, and D. M. de Leeuw, Unification of the Hole Transport in Polymeric Field-Effect Transistors and Light-Emitting Diodes, Phys. Rev. Lett. 91, 216601 (2003).

[32] C. Tanase, P. W. M. Blom, and D. M. de Leeuw, Origin of the enhanced space-charge-limited current in $\operatorname{poly}(p-$ phenylene vinylene), Phys. Rev. B 70, 193202 (2004).

[33] N. F. Mott and E. A. Davis, Electronic Processes in NonCrystalline Materials (Oxford University Press, Oxford, New York, 2012). Oxford Classic Texts in the Physical Sciences.

[34] F. Gao, A new acceptor for highly efficient organic solar cells. Joule 3, 908-909 (2019).

[35] J. C. Blakesley and D. Neher, Relationship between energetic disorder and open-circuit voltage in bulk heterojunction organic solar cells, Phys. Rev. B 84, 075210 (2011).

[36] Y. (Michael) Yang, W. Chen, L. Dou, W.-H. Chang, H.S. Duan, B. Bob, G. Li, and Y. Yang, High-performance multiple-donor bulk heterojunction solar cells, Nat Photon. 9, 190 (2015).

[37] P. Bi and X. Hao, Versatile ternary approach for novel organic solar cells: A review, Solar RRL 3, 1800263 (2019).

[38] N. Felekidis, E. Wang, and M. Kemerink, Open circuit voltage and efficiency in ternary organic photovoltaic blends, Energy Environ. Sci. 9, 257 (2015).

[39] N. Felekidis, A. Melianas, and M. Kemerink, Design rule for improved open-circuit voltage in binary and ternary organic solar cells, ACS Appl. Mater. Interfaces 9, 37070 (2017).

[40] H. Shi, R. Xia, G. Zhang, H.-L. Yip, and Y. Cao, Spectral engineering of semitransparent polymer solar cells for greenhouse applications, Adv. Energy Mater. 9, 1803438 (2019).

[41] G. Zuo, M. Linares, T. Upreti, and M. Kemerink, General rule for the energy of water-induced traps in organic semiconductors, Nat. Mater. 18, 588 (2019).

[42] L. J. A. Koster, Charge carrier mobility in disordered organic blends for photovoltaics, Phys. Rev. B 81, 205318 (2010).

[43] M. C. Heiber, K. Kister, A. Baumann, V. Dyakonov, C. Deibel, and T.-Q. Nguyen, Impact of tortuosity on chargecarrier transport in organic bulk heterojunction blends, Phys. Rev. Appl. 8, 054043 (2017).

[44] G. Zuo, Z. Li, O. Andersson, H. Abdalla, E. Wang, and M. Kemerink, Molecular doping and trap filling in organic semiconductor host-guest systems, J. Phys. Chem. C. 121, 7767 (2017).

[45] M. Nikolka, I. Nasrallah, B. Rose, M. K. Ravva, K. Broch, A. Sadhanala, D. Harkin, J. Charmet, M. Hurhangee, A. Brown, S. Illig, P. Too, J. Jongman, I. McCulloch, J.-L. Bredas, and H. Sirringhaus, High operational and environmental stability of high-mobility conjugated polymer fieldeffect transistors through the use of molecular additives, Nat Mater. 16, 356 (2016).

[46] H. van Eersel, R. A. J. Janssen, and M. Kemerink, Mechanism for efficient photoinduced charge separation at disordered organic heterointerfaces, Adv. Funct. Mater. 22, 2700 (2012).

[47] A. Melianas and M. Kemerink, Photogenerated charge transport in organic electronic materials: Experiments confirmed by simulations, Adv. Mater. 31, 1806004 (2019). 